\title{
NEFROLITIASIS: EVALUACIÓN METABÓLICA
} NEPHROLITHIASIS: METABOLIC EVALUATION

Natalia Sandí Ovares ${ }^{1}$ Natalia Salazar Campos² Carolina Mejía Arens³

1, 2 y 3 Médico general, Caja Costarricense del Seguro Social, San José Costa Rica

Contactos: nathalia.sandi1992@gmail.com natykatze@gmail.com carolinamejar17@gmail.com

\section{RESUMEN}

La nefrolitiasis es una patología urológica frecuente en la práctica médica, cuya incidencia ha aumentado en los últimos 20 años. Se clasifican principalmente según su composición química, en dos grandes grupos, los formados por calcio (oxalato de calcio y fosfato de calcio) y los no cálcicos (formados por ácido úrico, cistina y estruvita). Se han identificado como principales factores de riesgo para la formación de cálculos, la presencia de hiperparatiroidismo primario, acidosis tubular renal, factores dietarios y ciertos medicamentos. Las nuevas guías de manejo van dirigidas a la prevención, por lo que la evaluación metabólica para determinar el tipo de lito presente, se puede utilizar para guiar las recomendaciones terapéuticas de manejo médico conservador..

Palabras clave: Nefrolitiasis, oxalato de calcio, estruvita, urianálisis.

\section{Cómo citar:}

Sandí Ovares, N., Salazar Campos, N., \& Mejía Arens, C. (2021). Nefrolitiasis: evaluación metabólica. Revista Ciencia Y Salud, 5(1), Pág. 69-79.

\section{ABSTRACT}

Nephrolithiasis is a very common urologic pathology in medical practice and its incidence has increased in the last 20 years. The main classification is based on chemical composition in two large groups, those formed by calcium (calcium oxalate and calcium phosphate) and those that are not formed by calcium (uric acid, cystine and struvite). Some risk factors associated, to stone formation, are the presence of primary hyperparathyroidism, renal tubular acidosis, dietary factors and certain medications. The new management guidelines are aimed at prevention, so the metabolic evaluation is useful to determine the type of stone present, to guide therapeutic recommendations for conservative medical management.

Keywords: calcium oxalate, struvite, urinalysis, nephrolithiasis.

Recibido: $15 /$ dic/2020

Aceptado: 25/ene/2021

Publicado: $15 / \mathrm{feb} / 2021$

\section{(c) (1) (9)(2)}




\section{CIENCIA\&SALUD}

\section{INTRODUCCIÓN}

La nefrolitiasis es una de las patologías urológicas que se presenta con mayor frecuencia en la consulta externa y los servicios de emergencias. Tiene una incidencia de un $8.8 \%$ en los Estados Unidos, con tendencia al aumento, su fisiopatología se basa en la alteración de la solubilidad de los minerales que induce la cristalización (1, 2, 3).

En los últimos 20 años ha aumentado de forma considerable la incidencia de litiasis renal, la prevalencia de esta patología presenta un patrón geográfico, siendo la población caucásica la que más la presenta; también se encuentra relacionada con el género, se ha identificado que la mayoría de los casos son hombres entre los 40 y 60 años de edad $(1,3,5,6)$. Los cálculos de calcio son los más prevalentes en países desarrollados. Estudios demuestran que la recurrencia a los 5 años tras un primer episodio de litiasis cálcica es de un $50 \%(6,7)$.

El uso de uso de la litotripsia y la cirugía percutánea, como pilares de tratamiento de la nefrolitiasis, inicia en la década de los años ochenta; sin embargo, este tratamiento no logró disminuir la incidencia de la patología, ni mejorar la calidad de vida de los afectados (4).

De modo que el enfoque terapéutico fue redirigido, no a la curación de la enfermedad en sí, sino a la prevención de la formación de litos. El abordaje terapéutico propuesto inicia con definir el tipo de litiasis presente en el paciente, sus factores de riesgo y el riesgo de recurrencia de la enfermedad, para proporcionar una terapéutica individualizada según la fisiopatología de cada caso.

El objetivo principal de este artículo, es realizar una revisión bibliográfica actualizada centrada en el abordaje y estudio metabólico de la nefrolitiasis, que permita determinar el tipo de lito presente, para dirigir una estrategia de terapéutica conservadora individualizada, según el tipo de litiasis.

\section{MÉTODOS}

Se realizó un estudio descriptivo cualitativo de revisión bibliográfica de artículos obtenidos de las bases de datos de Pub med, utilizando como descriptores la palabra "nefrolitiasis" AND "oxalato de calcio" AND "manejo médico". Se seleccionó un total de 16 artículos en idioma inglés y español, publicados entre los años 2014 y 2019, que describen principalmente la fisiopatología, factores de riesgo, y manejo médico de nefrolitiasis. Entre ellos la mayoría corresponde a revisiones sistemáticas. Se excluyen aquellos artículos publicados en los años previos al 2014, los basados en la población adolescente y pediátrica, así como aquellos basados principalmente en el manejo quirúrgico.

\section{EPIDEMIOLOGIA}

En los últimos 20 años ha aumentado de forma considerable la incidencia de litiasis renal, siendo la población caucásica la más prevalente; también se encuentra relacionada con el género, se ha identificado que la mayoría de los casos son hombres entre los 40 y 60 años de edad $(1,3,5,6)$. Los cálculos de calcio son los más prevalentes en países desarrollados y la recurrencia a los 5 años tras un primer episodio de litiasis cálcica es de un $50 \%(6,7)$.

\section{FACTORES DE RIESGO}

El estudio transversal realizado por Arias R, et. al, describe los principales factores de riesgo asociados a nefrolitiasis en personas con edades entre los 45 y 65 años y determinó que la mayor incidencia se presenta en zonas cálidas, en personas de edad más avanzada, de clases sociales altas, con antecedentes familiares de nefrolitiasis, y comorbilidades como hipertensión arterial y sobrepeso (8).

Según Colditz, et. al, (9) se reportan como principales factores de riesgo, el antecedente heredo familiar de 


\section{CIENCIA\&SALUD}

nefrolitiasis, un índice de masa corporal (IMC) mayor a 35, ser mujer, enfermedad asociadas como diabetes mellitus tipo 2, hiperparatiroidismo primario, acidosis tubular renal, enfermedad de Crohn, gota, hipercolesterolemia o hipertrigliceridemia, factores dietéticos como la alta ingesta de oxalatos, de bebidas gaseosas y suplementos de vitamina $C$. Otros factores asociados son la baja ingesta de líquidos, el consumo frecuente de bebidas azucaradas, y un exceso de proteínas animales (5).

En los pacientes diabéticos, la presencia de un pH urinario bajo se ha asociado a mayor predisposición a formar de cristales de ácido úrico (1).

Los fármacos asociados a la formación de litos son: indinavir, aciclovir, sulfadiazina, metotrexate, triamtereno, diuréticos de asa, inhibidores de la anhidrasa carbónica, quinolonas, aminopenicilinas (9).

No existe evidencia contundente que demuestre que la ingesta de oxalato es un factor que aumenta el riesgo de nefrolitiasis. Respecto a la ingesta de calcio; algunos autores afirman su consumo, sí aumenta el riesgo de litiasis por calcio (1); mientras que otros mencionan que el aumento dietario de este elemento puede disminuir el riesgo, al unirse con oxalato a nivel intestinal, y disminuyendo su absorción (10).

Se ha determinado que el consumo de potasio disminuye la incidencia de formación de litos, ya que una alta ingesta de este elemento propicia su mayor unión con citrato, disminuyendo la precipitación del potasio y formación de litos (1).

En el caso de la hipopotasemia se ha visto que aumenta la absorción de citrato a nivel renal, lo que disminuye su excreción y aumenta la incidencia de litiasis. Mientras que ocurre lo contrario con las proteínas animales, a mayor ingesta, mayor cantidad de ácido úrico, que disminuye el pH urinario aumentando el riesgo de formación de litos de ácido úrico (10).

Como factores dietarios protectores se han asociado, la ingesta de leche, alta ingesta de frutas, fibra y vegetales (1).

\section{FISIOPATOLOGÍA}

Existen dos teorías con respecto a la formación de litos: la primera se basa en el descubrimiento en 1973 por Randall, quien sugirió que los depósitos de fosfato de calcio localizados en las papilas renales son la base ideal para la formación de litos de oxalato de calcio en las zonas denominadas placas de Randall, que se localizan en la base de la membrana del asa delgada de Henle, estas se encuentran en mayor cantidad en pacientes diabéticos, hipertensos y con aterosclerosis (10).

La segunda teoría asociada a la formación del litos es que cuando una sal se sobresatura en la orina precipita formando cristales, los cuales se fijan al urotelio y dan paso a la formación de litos (7).

Se ha descrito un orden cronológico en la formación de litos, inicialmente se produce saturación urinaria, posteriormente la supersaturación, la nucleación, el crecimiento de cristales, la agregación y retención de cristales y finalmente la formación del cálculo (7).

Para entender mejor este concepto, es importante tener claro que una sal puede disolverse en una solución hasta encontrar el equilibrio entre su fase acuosa y sólida a un $\mathrm{pH}$ y una temperatura determinada, la sobresaturación induce la cristalización, un proceso determinante para la formación de litiasis (2).

Existen inhibidores que impiden la formación de litos, entre estos destacan el citrato que presenta tanto un efecto inhibitorio en el crecimiento del lito como en la agregación de este. De manera similar, el magnesio puede inhibir la formación de litos por mecanismos aún no esclarecidos (10).

Se ha evidenciado que un pH mayor a 6, 7 altera la saturación y promueve la cristalización de fosfato de 


\section{CIENCIA\&SALUD}

calcio, lo cual explica el aumento en la incidencia en pacientes con acidosis tubular renal distal, hiperparatiroidismo primario y en pacientes que se encuentran en tratamiento con inhibidores de la anhidrasa carbónica. Por otra parte, ante la presencia de un $\mathrm{pH}$ acido $(<5,5)$ se produce la saturación de cristales de ácido úrico $(7,10)$.

\section{TIPOS DE LITOS SEGÚN SU COMPOSICIÓN QUÍMICA}

\section{Según la composición de los litos urinarios se clasifican en 2 grandes grupos (11):}

A. Litos compuestos por calcio: son los que se presentan en mayor frecuencia en la práctica clínica y se pueden dividir a su vez en cálculos de oxalato de calcio y fosfato de calcio.

B. Litos no compuestos por calcio: los cuales a su vez se dividen en tres grupos, cálculos de ácido úrico, cálculos de estruvita (formados por magnesio, amonio y fosfato) y los cálculos de cistina.

Las principales causas y características de los litos renales se describen en la Tabla 1.

Tabla 1. Etiología y características de los tipos de nefrolitiasis $(10,11)$.

Cálculos de sales de calcio

Disminución del volumen urinario

Volumen urinario menor a 1L/día aumenta el riesgo de nefrolitiasis

Hipercalciuria

Excreción de calcio mayor a $300 \mathrm{mg} / \mathrm{dL}$ en hombres

y mayor a $250 \mathrm{mg} / \mathrm{dL}$ en mujeres aumenta el riesgo de nefrolitiasis

Hiperoxaluria

Excreción de más de $40 \mathrm{mg} / \mathrm{dL}$ de oxalato de calcio (presente en un 10 a 50\% de las litiasis de calcio).

Hipocitraturia

Excreción de citrato disminuida (menor a 325mg/ $\mathrm{dL}$ ). Se ha asociado a una incidencia del 20 al $60 \%$ de litiasis de calcio.

Hiperuricosuria

Excreción urinaria de ácido úrico mayor a $750 \mathrm{mg} /$ $\mathrm{dL}$ en mujeres y mayor a $800 \mathrm{mg} / \mathrm{dL}$ en hombres,

disminuye la solubilidad del oxalato de calcio.

Cálculos no asociados a sales de calcio

Cálculos de ácido úrico (5 a 10\% de las litiasis renales)

Cálculos de fosfato de magnesio y amonio. (estruvita o carbonato de apatita)

Su formación se asocia principalmente a la precipitación de cristales de ácido úrico a un pH urinario menor a 5,5 (pH urinario de 5,5 mantiene el equilibrio del ácido úrico y el urato), el otro mecanismo de formación corresponde a la uricosuria, esta aumenta el riesgo de formación de cálculos con una excreción de ácido úrico en orina mayor a $800 \mathrm{mg} /$ día en hombres y mayor a $750 \mathrm{mg} /$ día en mujeres.

Ocurren consecuencia de la proliferación microbiana en asociación a la presencia de infecciones del tracto urinario, obstrucción del tracto de salida urinario, vejiga neurogénica, disfunción del vaciamiento, y catéteres urinarios. 


\section{CIENCIA\&SALUD}

Cálculos de cistina(1 a 2\% de las litiasis renales)

Litiasis de origen genético, asociada a un patrón de herencia autosómica recesiva, que ocasiona un defecto en el transportador de aminoácidos expresado en el túbulo proximal renal, lo que ocasiona la formación de cristales hexagonales (hallazgo patognomónico), cuando los niveles de cistatina son mayores a $250 \mathrm{mg} / \mathrm{L}$. También se pueden formar cristales por uso de ampicilina o en pacientes con anemia de fanconi.

Fuente: Elaboración propia de autoras, basado en el artículo publicado por Pfau A \& Knauf F (10, 11).

\section{ESTUDIOS DIAGNÓSTICOS}

Es importante definir que el diagnóstico de nefrolitiasis es clínico: dolor agudo, de tipo cólico en el flanco ipsilateral, que no alivia con cambios de posición, si el dolor es constante se asocia en mayor medida a una obstrucción severa. Esta clínica se puede acompañar de hematuria macroscópica o microscópica, náuseas o vómitos (1).

Dentro de las técnicas de imagen diagnosticadas, destacan las siguientes $(6,7)$ :

- $\quad$ Radiografía de Tórax: constituye la prueba más rápida, y accesible; con una sensibilidad del $45 \%$ y una especificidad de $77 \%$ en detección de litos formados por calcio.

- $\quad$ Ultrasonido: esta técnica tiene la ventaja de que permite evitar la exposición radiológica innecesaria, se recomienda el ultrasonido en el paciente pediátrico y en mujeres embarazadas con sospecha de nefrolitiasis, tiene una sensibilidad del $61 \%$ y una especificidad del $97 \%$; sin embargo, usualmente sobrestima el tamaño del lito en aproximadamente 2 milímetros.

- Tomografía axial computarizada con medio de contraste: es el método Gold Standard para el diagnóstico.

- Resonancia magnética: se recomendada en pacientes embarazadas en el primer trimestre con un ultrasonido dudoso.

\section{EVALUACIÓN METABÓLICA}

La Asociación Americana de Urología (AAU) recomienda realizar como parte del abordaje diagnóstico una valoración metabólica, que incluya una orina de 24 horas, con análisis completo de electrolitos, química sanguínea completa y urianálisis, especialmente en pacientes con un primer episodio asociado a factores riesgo, la formación de cálculos recurrentes, pacientes monorrenos, en población pediátrica o adultos jóvenes y la ante la presencia de múltiples litos en un primer episodio $(12,13)$.

Aportes de cada estudio para el diagnóstico $(12,14)$ :

1. Química Sanguínea: importante para valorar electrolitos, ya que un nivel sérico potasio bajo acompañado de cloruro elevado y pH urinario mayor a 6,5 sugieren la presencia de acidosis tubular renal de tipo 1. Otro electrolito esencial a valorar es el calcio, cuando existen niveles altos o normales altos ( $\geq 10 \mathrm{mg} / \mathrm{dL}$ ) se deben valorar los niveles de hormonas paratiroideas. Niveles altos de ácido úrico pueden indicar gota, aunque solo un 20 \% de los pacientes con gota van a desarrollar cálculos de ácido úrico. Además, se debe valorar la glicemia, y el perfil de lípidos en pacientes obesos debido a la alta prevalencia de la litiasis renal en personas con síndrome metabólico.

2. Urianálisis: es importante determinar el pH urinario, ya que los litos de ácido úrico solo se forman a $\mathrm{pH}$ menor o igual a 5,5, mientras que los de fosfato de calcio y estruvita requieren un $\mathrm{pH}$ mayor a 7,25. El reporte microscópico puede dar más información sobre la morfología de los cristales (cristales hexagonales 


\section{CIENCIA\&SSALUD}

son patognomónicos de los cálculos cistina). La tira reactiva al igual que la microscopia pueden orientar a cerca de datos de infección urinaria, que predisponen a cálculos de estruvita.

3. Orina 24 horas: se deben tomar de 1-2 muestras, cada muestra debe ser recolectada manteniendo una dieta normal y en el reporte se debe incluir los valores del volumen urinario total, pH, calcio, oxalato, ácido úrico, citrato, sodio, potasio y creatinina, datos necesarios para determinar la composición de los litos, y la presencia de inhibidores o promotores de la formación de litios.

Algoritmo diagnóstico de la composición litiásica

Clayman et. al, (14) describen un algoritmo diagnóstico y otro algoritmo terapéutico, de abordaje de la nefrolitiasis, que denominan "Stone tres", (ver figura 1 y 2 ).

En el caso de la nefrolitiasis por calcio, la clave es valorar el calcio urinario en el contexto de oxalato de calcio (tipo de cálculo más común) se proponen el siguiente abordaje:

A. $\quad$ Si existe normocalciuria, se describen solamente tres etiologías posibles (1):

1. Litiasis por exceso de promotor, son principalmente litos de ácido úrico.

2. Litiasis por inhibidor insuficiente, se asocia a bajos niveles de citrato.

3. Litiasis por exceso soluto, se asocia a hiperoxaluria, si la excreción urinaria de oxalato de calcio es mayor a $75 \mathrm{mg} /$ día, se considera primaria y si es menor a $75 \mathrm{mg} /$ día, se deben considerar problemas de absorción o dietéticos.

B. Si existe hipercalciuria, inicialmente se deben determinar los niveles séricos de calcio, y según estos se procede a clasificar la etiología (14).

1. Con calcio sérico elevado, se deben valorar los niveles de parathormona (PTH).

Si el nivel de PTH se encuentra elevado, se puede considerar una litiasis por hiperparatiroidismo primario.

- $\quad$ Si el nivel de PTH se encuentra suprimido, se debe considerar sarcoidosis.

2. Con calcio sérico normal, no se recomienda realizar la prueba de carga de calcio como se hacía anteriormente para distinguir, hipercalciuria renal de hipercalciuria absortiva, ya que, sin importar la etiología, el manejo es el mismo. En este tipo pacientes se recomienda realizar una densitometría ósea debido a la alta incidencia de osteoporosis y osteopenia (5).

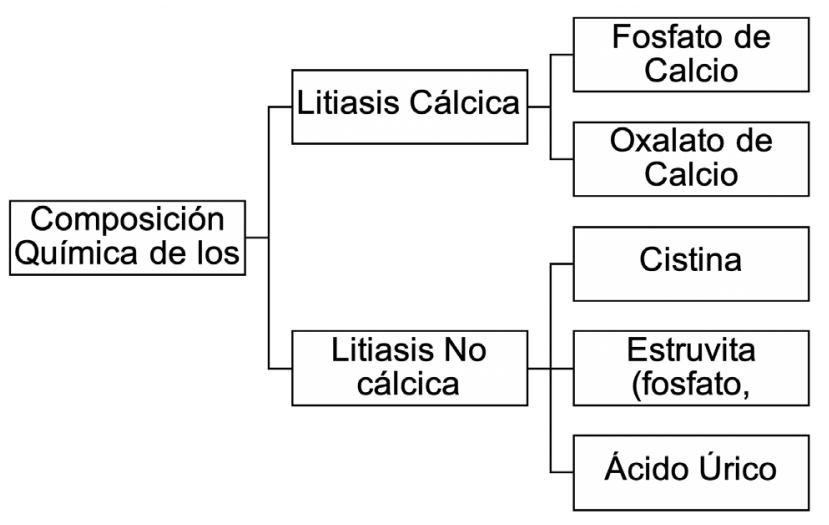

Figura 1. Clasificación según composición química de los litos urinarios (14). Elaboración propia de autoras, basado en Clayman R, et al.

En el caso de la nefrolitiasis no cálcica, existen tres etiologías posibles, y cada una de ellas se asocia a la 


\section{CIENCIA\&SALUD}

formación de un tipo de lito $(4,14)$ :

1. Cálculos de ácido úrico: necesita un $\mathrm{pH}$ menor a 5,5 y puede acompañarse de uricosuria.

2. Cálculos de estruvita: necesita un pH mayor a 7,25, se presenta en cuadros de infecciones a repetición por bacterias que tienen la enzima ureasa (Proteus, Klebsiella, Pseudomonas), que alcalinizan la orina y predisponen a la formación de cálculos de magnesio-amonio-fosfato.

3. Cálculos de cistina: necesita un pH menor a 7,0 y niveles de cistina urinaria elevados.

\section{MANEJO MÉDICO}

Aumento del volumen urinario: todos los tipos de cálculos se asocian a concentraciones urinarias elevadas de sales específicas por lo que, para disminuir la concentración de sales urinarias, se recomienda un consumo de líquidos que logre alcanzar un volumen urinario mayor a 2.5 L/día (3).

Intervenciones dietéticas: actualmente se recomienda en pacientes con urolitiasis cálcica e hipercalciuria una dieta con consumo diario de 1000-1200 mg/día de calcio (2). Un consumo alto en calcio reduce el riesgo de nefrolitiasis, en comparación con una dieta con un bajo consumo (10). Esto debido a que el consumo alto de calcio disminuye la absorción y excreción del oxalato (14). El sodio por su parte, aumenta la excreción urinaria de calcio, por lo que se recomienda una dieta baja en sodio (3). Otras especificaciones en la dieta se describen más adelante en la sección de manejo según tipo de cálculo urinario (7, 14). Las frutas y vegetales proveen una carga alcalina que aumenta el citrato urinario por lo que se recomienda su consumo para aumentar el aporte de citrato en la dieta, mientras que las proteínas de origen animal aumentan la carga ácida urinaria, disminuyendo el citrato urinario, por lo que se recomienda mantener una dieta baja en proteínas de origen animal (7). Se ha demostrado que el jugo de uva aumenta el riesgo de nefrolitiasis, mientras que el consumo de leche lo disminuye; con respecto al consumo de soda o cafeína no se ha evidenciado que represente un riesgo en la formación de nefrolitiasis, mientras que el alto consumo de vitamina $C$ se asocia a mayor riesgo de nefrolitiasis por oxalato de calcio por lo que no se recomienda un exceso de suplementación con vitamina C (1).

\section{FARMACOTERAPIA:}

Los pacientes con litos compuestos por oxalato de calcio deben recibir tratamiento farmacológico de acuerdo con el mecanismo de formación de litos asociado:

Hiperuricosuria (exceso de promotor): manejo inicial con dieta baja en purinas y proteínas. Si la dieta falla, se agrega alopurinol iniciando con $100 \mathrm{mg} /$ día y se aumenta progresivamente hasta $300 \mathrm{mg} /$ día (14).

Niveles de citrato bajos (inhibidor bajo): la terapia consiste en una dieta alta en álcali proveniente de frutas y vegetales, la dieta por sí sola tiene un efecto muy leve por lo que se acompaña de fármacos alcalinizantes $(15,16)$, el fármaco más utilizado es el citrato de potasio, el cual se encuentra en dos formas: tabletas de citrato de potasio (dosis 10-20 mEq dos veces al día) o citrato potasio liquido (30 mEq de dos a tres veces al día), otras opciones terapéuticas son el citrato de sodio o bicarbonato de sodio (5).

Hiperoxaluria (exceso soluto): su manejo consiste en una dieta baja en oxalatos (espinacas, nueces, papas, chocolate) y un alto consumo de calcio, ya que la hipercalcemia disminuye la absorción y excreción de oxalato. Se recomiendan suplementos de calcio debido a la mala absorción de este en el organismo (14, 16).

Hipercalciuria: podemos tener pacientes con calcio sérico alto y bajo, por lo tanto:

Hipercalcemia: en combinación con niveles séricos de PTH elevada lleva al diagnóstico de hiperparatiroidismo. La causa más común es el adenoma seguido de hiperplasia. El manejo es quirúrgico, una paratiroidectomía (3). 


\section{CIENCIA\&SALUD}

Normocalcemia: de etiología es variable, puede ser por mayor absorción intestinal o mayor pérdida a nivel renal. Independientemente de la etiología, el manejo es con diuréticos tipo tiazidas, y se prefiere la clortalidona a la hidroclorotizida (3).

Idiopáticos: pacientes con litiasis cálcica en los que no se ha encontrado ninguna anormalidad metabólica. Estos se manejan empíricamente con diuréticos tiazida y / o citrato de potasio. Estas terapias han demostrado prevenir la recurrencia pesar de su origen metabólico desconocido (5).

Los pacientes con litos no cálcicos deben recibir el tratamiento dependiendo del tipo de lito presente:

Cálculos de cistina: se debe alcanzar una meta de concentración urinaria de cisteína menor a 250 $\mathrm{mg} / \mathrm{L}$, esto se puede lograr con la ingesta de tres a cuatro litros de líquidos diarios, una baja ingesta de sodio y proteínas además de tratamiento farmacológico. Las proteínas de origen animal son ricas en cistina y metionina, esta última es metabolizada en a-cistina y aumenta la excreción urinaria de sodio $(4,7,15)$. La solubilidad de cistina aumenta a pH ácido, por lo que son útiles opciones farmacológicas para alcalinizar el $\mathrm{pH}$ con citrato de potasio (13). Si hay falla terapéutica, existen opciones como tioles que se unen a la cistina para permitir su solubilidad en agua, en este grupo se encuentran la tiopronina, D-penicilamina y captopril. La tiopronina es el agente preferido debido a su alta eficacia (7).

- Cálculos de estruvita: el objetivo es eliminar las bacterias que contienen la enzima ureasa (Proteus, Enterococcus, Pseudomona, Klebsiella Escherichia coli), por lo que remover los cálculos es esencial para eliminar el nido bacteriano y así evitar la recurrencia. Se recomienda tratamiento antibiótico con cefalosporinas de primera y segunda generación, tanto dos semanas antes, como seis a doce meses después de la remoción quirúrgica de los cálculos, y realizar un control con urocultivo a los tres, seis y doce meses $(2,11$, 14)

. En pacientes con alto riesgo de recurrencia o aquellos que no se puedan someter a la extracción quirúrgica de los cálculos se puede recurrir a bloqueadores de ureasa, como el ácido acetohidroxámico (250 mg TID o hasta un máximo de 1,5 g/día); un potente inhibidor de la ureasa, sin embargo, presenta pobre adherencia, ya que presenta efectos adversos severos, tales como flebitis y fenómenos tromboembólicos, por lo que ante la poca tolerabilidad de los pacientes al tratamiento médico, se prefiere el manejo quirúrgico $(2,10)$.

- Cálculos de ácido úrico: como manejo inicial se propone una dieta baja en purinas y con gran contenido alcalino, desafortunadamente la evidencia de mejoría clínica con dieta es escasa. La clave consiste en mejorar el pH urinario con citrato de potasio, alcanzando una meta de pH entre 6,0 y $6,5(14,15)$. El alopurinol no debe ser tratamiento de primera línea debido a que la principal anormalidad metabólica es el pH bajo y no la hiperuricosuria. Si la alcalinización no previene la recurrencia, o hay una excreción urinaria elevada de ácido úrico, el alopurinol sí estaría indicado $(7,15)$.

Para el seguimiento del tratamiento de todos los tipos de cálculos se recomienda un monitoreo periódico de química sanguínea. La orina de 24 horas se repite en un período de entre seis semanas a cuatro meses después del inicio del tratamiento médico y posteriormente en un intervalo menos frecuente según la evolución del paciente. Los estudios de imágenes se repiten a los 6 meses del inicio del tratamiento y posteriormente, tan pronto como sea necesario, según los resultados de la orina de 24 horas control (14). 


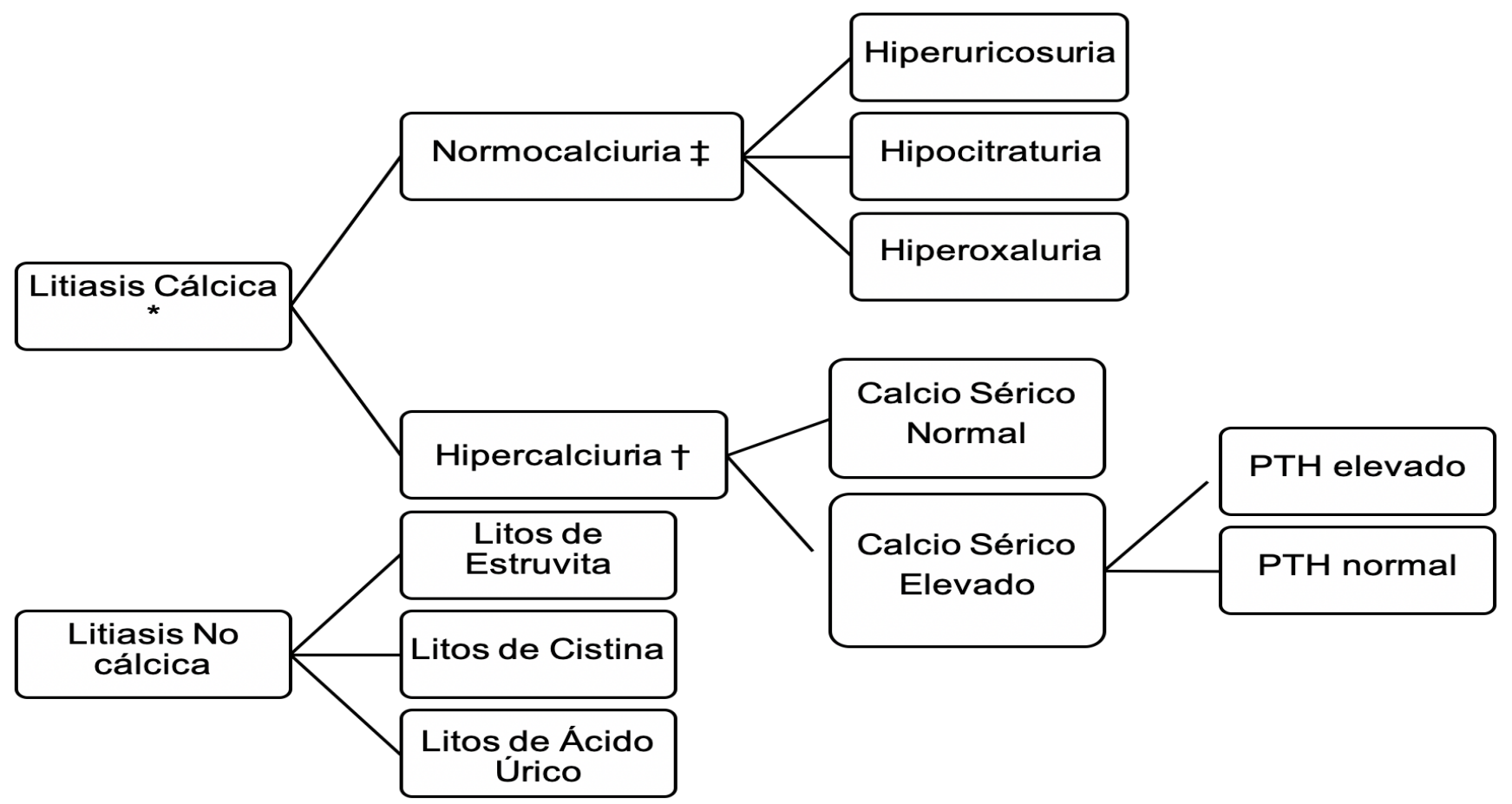

Figura 2. Algoritmo diagnóstico simplificado (14). *Se presume que es oxalato de calcio debido a su frecuencia. $\ddagger$ En caso de normo calciuria se busca un exceso de promotor, nivel bajo de inhibidor o exceso de soluto †. Se recomienda realizar densitometría ósea por riesgo osteoporosis. Elaboración propia de autoras, basado en Clayman R, et al.

\section{CONCLUSIONES}

La incidencia de la nefrolitiasis ha aumentado en los últimos años, asociada a los cambios en el estilo de vida y dietéticos. Su abordaje mediante cirugía percutánea no ha demostrado disminuir la incidencia de la patología, ni mejorar la calidad de vida, por lo que se describe una nueva estrategia terapéutica, basada en la determinación del mecanismo patológico subyacente.

Se recomienda como parte del abordaje diagnóstico una valoración metabólica, que incluya orina de 24 horas, análisis de electrolitos, química sanguínea completa y urianálisis, especialmente en pacientes con factores riesgo, asociados a alta recurrencia.

Mediante el análisis metabólico se determina el tipo de cálculo presente y se dirige un tratamiento médico preventivo, que ha demostrado efectividad comparada al tratamiento quirúrgico, sin las complicaciones asociadas a éste. Este tratamiento se enfoca según el tipo de cálculo presente, e incluye recomendaciones dietéticas y en algunos casos terapia farmacológica. 


\section{CIENCIA\&SALUD}

\section{REFERENCIAS BIBLIOGRÁFICAS}

1. Mayans L. Nephrolithiasis. Primary Care: Clinics in Office Practice. [Internet]. 2019; [citado: 20 de febrero 2020]; 46(2), 203-212. Disponible en: https://doi.org/10.1016/j.pop.2019.02.001

2. Ludwig, W. W., \& Matlaga, B. R. Urinary Stone Disease. Medical Clinics of North America, [Internet]. 2018; [citado: 20 de febrero 2020]; 102(2), 265-277. Disponible en: https://doi.org/10.1016/j. mcna.2017.10.004

3. Sorokin I, Mamoulakis C, Miyazawa K, Rodgers A, Talati J, Lotan Y. Epidemiology of stone disease across the world. World Journal of Urology. [Internet]. 2017; [citado: 20 de febrero 2020]; 35(9): 1301-1320. Disponible en: https://doi.org/10.1007/s00345-017-2008-6

4. Peña Rodríguez José Carlos. Avances y retos en la fisiopatología y tratamiento de la nefrolitiasis. Acta méd. Grupo Ángeles [revista de Internet]. 2016, [citado: 9 de marzo de 2020]; 14(3):155-161. Disponible en: http://www.scielo.org.mx/scielo.php?script=sci_arttext\&pid=S1870-72032016000300155\&lng=es

5. Susaeta R, Benavente D, Marchant F, Gana R. Diagnóstico y manejo de litiasis renales en adultos y niños. Revista Médica Clínica Las Condes. [Internet]. 2018; [citado: 20 de febrero 2020]; 29(2):197-212. Disponible en: doi: 10.1016/j.rmclc.2018.03.002.

6. Nicolau C, Salvador R, Artigas J, Manejo diagnostico de cólico renal. Radiologia. [Internet]. 2015; [citado: 20 de febrero 2020]; 57(2): 113-122. Disponible en: Doi: https://doi.org/10.1016/j.rx.2014.11.003

7. García-Perdomo, H. A., Solarte, P. B., \& España, P. P. Fisiopatología asociada a la formación de cálculos en la vía urinaria. Urología Colombiana, [Internet]. 2016; [citado: 20 de febrero 2020]; 25(2):109-117. Disponible en: doi:10.1016/j.uroco.2015.12.012

8. Arias Vega R, Pérula de Torres L, Jiménez C, Carrasco J, Requena M, Cano R, Silva L. Comorbilidad y factores sociodemográficos asociados a litiasis renal en personas de 40 a 65 años: estudio transversal. Medicina clínica. [Internet]. 2017; [citado: 20 de febrero 2020]; 149(9):383-390. Disponible en: https://doi. org/10.1016/j.medcle.2017.09.017

9. Colditz G, Philpott S, Hankinson S. The Impact of the Nurses' Health Study on Population Health: Prevention, Translation, and Control. American Journal Of Public Health, [Internet]. 2016; [citado: 20 de febrero 2020]; 106(9):1540-1545. Disponible en: doi: 10.2105/ajph.2016.303343

10. Pfau A, Knauf F. Update on Nephrolithiasis: Core Curriculum 2016. American Journal Of Kidney Diseases, [Internet]. 2016; [citado: 20 de febrero 2020]; 68(6):973-985. Disponible en: doi: 10.1053/j. ajkd.2016.05.016

11. Sorokin I, Pearle M. Medical therapy for nephrolithiasis: State of the art. Asian Journal of Urology. [Internet]. 2018; [citado: 20 de febrero 2020]; 5: 243-55. Disponible en: doi: 10.1016/j.ajur.2018.08.005

12. Goharderakhshan R, NG C, Guerrero C, Loo R. Mp95-15 population management kidney stone care approach improves compliance with the American Urological Association (AUA) recommendations on medical management of kidney stones. Journal Of Urology, [Internet]. 2017; [citado: 20 de febrero 2020]; 197(4S). Disponible en: doi: 10.1016/j.juro.2017.02.3017

13. Tiselius G, Daudon M; Thomas K, Seitz C. Metabolic Work-up of Patients with Urolithiasis: Indications and Diagnostic Algorithm. European Urology Focus. [Internet]. 2017; [citado: 20 de febrero 2020]; 3(1):6271. Disponible en: https://doi.org/10.1016/j.euf.2017.03.014 


\section{CIENCIA\&SALUD}

14. Clayman R, Patel R, Pearle M. "STONE TREES": Metabolic Evaluation and Medical Treatment of the Urolithiasis Patient Made Easy. Journal of Endourology, [Internet]. 2018; [citado: 20 de febrero 2020]; 32(5):387-392. Disponible en: https://doi.org/10.1089/end.2017.0541

15. Dion M, Ankawi G, Chew B, Paterson R, Sultan N, Hoddinott P, Razvi, H. CUA guideline on the evaluation and medical management of the kidney stone patient - 2016 update. Canadian Urological Association Journal. [Internet]. 2016; [citado: 20 de febrero 2020]; 10(11-12): E347-58. Disponible en: https://doi. org/10.5489/cuaj.4218

16. García V, Yanes I, Tejera P, Perez G, Moraleda T. The idiopathic hypercalciuria reviewed. Metabolic abnormality or disease?. Nefrologia. [Internet]. 2019; [citado: 20 de febrero 2020]; 39(6):592-602. Disponible en: doi: 10.1016/j.nefro.2019.02.011. 